\title{
Aprendizaje para la preparación de oposiciones de acceso a la carrera judicial y fiscal en el Grado de Derecho de la Universidad Europea de Valencia
}

Carla de Paredes Gallardo a

a Profesora de la Universidad Europea de Valencia, España.

\begin{abstract}
Resumen
La universidad Europea de Valencia ha introducido como innovación docente en la metodología del grado de Derecho la preparación de oposiciones para aquellos estudiantes que tras finalizar sus estudios ante un mercado tan competitivo deciden optar por las oposiciones por ello se pretende que los estudios universitario les permita aprovechar como preparación para las oposiciones haciendo uso de técnicas de estudio y de técnicas orales con el fin de que les resulte más fácil.

Se aplica en la asignatura de Derecho de Familia y Sucesiones con 6 E.C.T.S, que se cursa en el tercer curso del Grado de Derecho en la Universidad Europea de Valencia durante el curso académico 2014/2015 y 2015/2016, con un total de 25 estudiantes, introduciendo unas jornadas de "técnicas de estudio y de expresión oral" con el fin de que los estudiantes adquieran agilidad y fluidez en el aprendizaje del temario.

Finalmente se realiza la prueba de conocimiento final de la asignatura de manera oral simulando un ejercicio real de la oposición para que el estudiante vaya adquiriendo preparación y experiencia.
\end{abstract}

Palabras claves: Oposiciones, estudio, comunicación oral.

\section{Introducción}

La preparación de oposiciones de acceso a la Carrera Judicial y Fiscal es una alternativa profesional elegida por muchos graduados tras finalizar sus estudios del Grado de Derecho.

Tradicionalmente, muchos de estos graduados accedían a esta alternativa sin ninguna preparación ni conocimiento de cómo afrontar esta nueva etapa.

La preparación del examen de oposición, no es solo es una cuestión de conocimientos teóricos, sino que también implica otras muchas competencias:

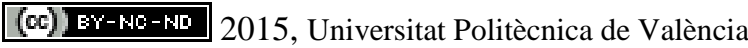

Congreso In-Red (2015) 
Aprendizaje para la preparación de oposiciones de acceso a la carrera judicial y fiscal en el Grado de Derecho de la Universidad Europea de Valencia

- $\quad$ Pautas de planificación del trabajo.

- $\quad$ Organización del tiempo de estudio por parte del graduado.

- $\quad$ Entrenamientos con técnicas para evitar o disminuir el stress el día del examen y los días previos al mismo.

- Preparación de técnicas de concentración, autoestima y relajación.

- Preparación con técnicas para evitar el “bloqueo” el día del examen.

- Pautas para saber actuar y comportarse.

- $\quad$ Pautas para saber dirigirse al Tribunal de la oposición.

Debido a esta carencia en años anteriores, el Grado de Derecho de la Universidad Europea de Valencia ha introducido unas jornadas para orientar al estudiante en el mundo de las oposiciones. De esta manera, el estudiante indeciso puede descartar esta opción por la complejidad de las mismas y optar por otra elección sin que ello implique una pérdida de posibilidades en su carrera profesional.

Toda esta metodología permite al estudiante que decide seguir por el camino de la preparación de las oposiciones ganar tiempo aprovechando los años de estudio del Grado.

\section{Objetivos}

El objetivo del presente trabajo son los que se enumeran a continuación:

1. Valorar las ventajas que supone introducir en los estudios de Grado de Derecho metodologías que orienten al estudiante para la preparación del futuro examen de oposiciones, tanto de Notarias y Registro como para el acceso a la Carrera Judicial y Fiscal, permitiendo al estudiante la adquisición no solo de los conocimientos jurídicos necesarios para la superación de las pruebas orales sino también de otros recursos o competencias necesarias y útiles para poder hacer más eficaz el estudio de las oposiciones.

2. Por otro lado se pretende poner de relieve la necesidad de trabajar otras técnicas, como la comunicativa, la expresiva, la relajación, igual de importantes y necesarias que las técnicas de adquisición de conocimientos para la preparación y superación de las pruebas, ya que permiten usar herramientas necesarias para poder manifestar los conocimientos jurídicos que durante tanto tiempo se han trabajado. Por todo ello, si tanto las técnicas de conocimientos jurídicos como las técnicas comunicativas, expresiva son trabajados durante el estudio del Grado de Derecho, al finalizar el mismo, dichas técnicas, las jurídicas y las expresivas, están ya muy trabajadas lo que permiten ganar agilidad, tiempo y eficacia en la preparación de las oposiciones 
y poder aprobar el examen en menor tiempo y principalmente lograr la motivación del estudiante.

3. Conocer la opinión de los estudiantes respecto a la aplicación de esta nueva metodología de innovación docente mediante la encuesta de valoración personal.

\section{Desarrollo}

\section{Jornadas de "Técnicas de estudio y de expresión oral"}

En primer lugar y para llevar a cabo esta metodología docente, se han introducido en el $1^{\circ}$ trimestre de $3^{\circ}$ curso del Grado de Derecho de la Universidad Europea de Valencia, en el año académico 2013/2014 y 2014/2015 y en asociación con la asignatura de "Derecho de Familia y Sucesiones" con 6 E.C.T.S, con un total de 25 estudiantes, unas jornadas denominadas "Técnicas de estudio” y “Técnicas de expresión oral”.

Estas jornadas desarrollan una serie de competencias para proporcionar al estudiante unas pautas necesarias para afrontar mejor el estudio de las oposiciones, como en la planificación del trabajo, la organización del tiempo a la hora de exponer el tema, la realización de técnicas para evitar o disminuir el stress, o evitar el bloqueo ante el Tribunal mediante técnicas de respiración, la preparación de técnicas de concentración, de autoestima y relajación.

Para que los estudiantes adquieran agilidad, fluidez en el aprendizaje del temario y un adecuado uso del vocabulario jurídico, el contenido de dicha "oposición ficticia” es el temario de la asignatura "Derecho de Familia y Sucesiones".

\section{Actividades complementarias.}

Previamente y durante el trimestre, se ha concertado una actividad fuera de la Universidad para que los estudiantes realicen una visita al Colegio de Registradores y Notarios de Valencia, para poder presenciar en directo un "simulacro de examen por un opositor" ante un Tribunal formado por distintos Notarios y Registradores de Valencia.

\section{La prueba final de conocimiento oral}

La prueba final de conocimiento de la asignatura Asimismo se les facilita la posibilidad de realizar la prueba de conocimiento de la asignatura de manera oral simulando un ejercicio real de la oposición para que vayan adquiriendo preparación y experiencia.

Esta prueba final de conocimiento de la asignatura consiste en la exposición oral de los temas de la asignatura ciñéndose al tiempo reglamentario de 15 minutos por tema y citando literalmente los artículos contenidos en el tema correspondiente.

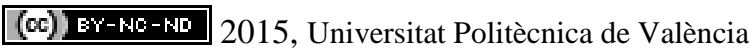


Aprendizaje para la preparación de oposiciones de acceso a la carrera judicial y fiscal en el Grado de Derecho de la Universidad Europea de Valencia

La elección de los temas para la exposición oral se realiza por el mismo estudiante eligiendo tres temas de manera aleatorias, siguiendo la misma metodología que en un examen de oposición real, es decir, el estudiante elige una bola de plástico de dentro de una bolsa de tela opaca representando cada bola cada uno de los temas de la asignatura.

Los temas deben ser expuestos ante un Tribunal, está formado por profesores del Claustro de Ciencias Sociales de la Universidad Europea de Valencia. El resto de los estudiantes matriculados en la asignatura, deberán asistir como público tal y como se realiza en un examen real de oposición.

La nota del examen cuenta un $40 \%$ de la nota final de la asignatura de Derecho de Familia y Derecho de Sucesiones, el $60 \%$ del resto de la nota de la asignatura se obtiene con la realización de trabajos en el aula.

\section{Encuesta de Valoración por parte del estudiante.}

En segundo lugar, se ha desarrollado una encuesta de valoración de la opinión de los estudiantes respecto dicho seminario.

La encuesta estaba formada por 10 preguntas, se adjunta como anexo I.

\section{Resultados}

Los resultados de la metodología de aprendizaje utilizados han consistido únicamente en una encuesta de valoración de la opinión de los estudiantes de manera oral respecto a las técnicas de estudio y las técnicas de expresión oral aplicadas al conocimiento jurídico, mostrando una gran satisfacción por parte de los graduados ya que han adquirido una gran fluidez a la hora de cantar los temas evitando la improvisación para evitar bloqueos en la exposición , un gran vocabulario jurídico y una rapidez a la hora del estudio de la materia el trabajo en la oposición.

Algunos estudiantes manifestaron que esta metodología les sirvió para descartar la preparación de oposiciones como posible elección una vez terminado el grado, permitiéndoles la posibilidad de buscar otras opciones.

Fuera del ámbito jurídico, manifiestan que han aprendido la importancia de mantener una actitud positiva durante el estudio, así como el saber estar y como dirigirse ante el Tribunal, cuestiones también relevantes en la preparación de una oposición.

En este mismo sentido, el profesor observa una evolución importante de los estudiantes en la forma de expresarse desde el comienzo del trimestre hasta la fecha de realización del examen donde ponen en práctica todas las técnicas explicadas en las jornadas.

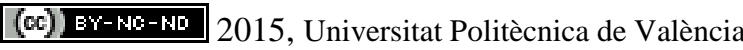

Congreso IN-RED (2015) 


\section{Conclusiones}

La conclusión que se llega con la aplicación de esta innovación docente novedosa es que los estudiantes del Grado de Derecho se familiarizan mucho con el temario y con las técnicas de estudio para la preparación de oposiciones de Notarias, Registro y al acceso a la Carrera Judicial y Fiscal, consiguiendo ganar tiempo en la preparación de las oposiciones.

Les permite tener una preparación respecto técnicas de estudio, oratoria y concentración que de otra manera no hubieran adquirido hasta llevar un año en la preparación de oposiciones.

Desde el punto de vista del profesor se observa una evolución en la manera de expresarse y una madurez jurídica que sin aplicación de las técnicas de oralidad, expresión y relajación no se lograban.

El inconveniente principal de esta metodología es el reflejo de la dureza de las oposiciones y con ello la desilusión de algunos estudiantes respecto a comenzar a estudiar una oposición.

\section{Referencias}

BAGUES, M.F. (2005). “¿Qué determina el éxito en unas oposiciones?”. Universidad de Islas Baleares. XII Encuentro de Economía Pública: Evaluación de las Políticas Públicas. Palma de Mallorca. Pág. 13.

BAGÜES, M.F. (2007). "Las oposiciones: análisis estadístico”. Jueces para la democracia, $\mathrm{n}^{\circ} 59$,

FELGUEROSO FERNANDEZ, F., PEREZ VILLAdONIGA, M.J., BAGÜES, M.F., (2007). "Sobre la composición optima de los tribunales evaluadores: el caso de las oposiciones a Jueces y Fiscales”. Cuadernos económicos de ICE. Número 74. Páginas 147166.

SAIZ ARNAIZ, A., (2012). “La selección de los jueces en España: la oposición”. Revista del poder judicial. Número 93.

CGPJ. $\quad$ http://www.poderjudicial.es/cgpj/es/Servicios/Acceso-a-la-Carrera-Judicial-Jueces-y-Fiscales. (Consulta 1 de abril de 2015) 
Aprendizaje para la preparación de oposiciones de acceso a la carrera judicial y fiscal en el Grado de Derecho de la Universidad Europea de Valencia

\section{ANEXO I.}

ENCUESTA DE SATISFACCION. Marca una de las casillas para cada pregunta

1. ¿ Te han facilitado las jornadas de técnicas de estudio alguna herramienta que no conocías?

Muy de acuerdo $\square \quad$ De acuerdo $\square \quad$ Indiferente $\square \quad$ En desacuerdo $\square \quad$ Muy en desacuerdo $\square$

2. ¿ Te han ayudado las técnicas de expresión oral en la exposición de los temas?.

Muy de acuerdo $\square \quad$ De acuerdo $\square \quad$ Indiferente $\square \quad$ En desacuerdo $\square \quad$ Muy en desacuerdo $\square$

3. ¿Crees que los contenidos aprendidos son de utilidad práctica?.

Muy de acuerdo $\square \quad$ De acuerdo $\square \quad$ Indiferente $\square \quad$ En desacuerdo $\square \quad$ Muy en desacuerdo $\square$

4. ¿Los posibles problemas que hayan surgido durante el proceso de enseñanza-aprendizaje, se han resuelto con eficacia?.

Muy de acuerdo $\square \quad$ De acuerdo $\square \quad$ Indiferente $\square \quad$ En desacuerdo $\square \quad$ Muy en desacuerdo $\square$

5. ¿Estás satisfecho con el desarrollo del proceso enseñanza-aprendizaje en las jornadas?.

Muy de acuerdo $\square \quad$ De acuerdo $\square \quad$ Indiferente $\square \quad$ En desacuerdo $\square \quad$ Muy en desacuerdo $\square$

6. ¿Te ha permitido la realización del examen de la asignatura de manera oral, adquirir conocimientos y otras habilidades?.

Muy de acuerdo $\square \quad$ De acuerdo $\square \quad$ Indiferente $\square \quad$ En desacuerdo $\square \quad$ Muy en desacuerdo $\square$

7. ¿Estas satisfecho con la realización de las jornadas?

Muy de acuerdo $\square \quad$ De acuerdo $\square \quad$ Indiferente $\square \quad$ En desacuerdo $\square \quad$ Muy en desacuerdo $\square$

8. En tu opinión, ¿̇se ha conseguido alcanzar los objetivos previstos en la asignatura?.

Muy de acuerdo $\square \quad$ De acuerdo $\square \quad$ Indiferente $\square \quad$ En desacuerdo $\square \quad$ Muy en desacuerdo $\square$

9. ¿Recomendaría las jornadas y la metodología a otro estudiante?.

Muy de acuerdo $\square \quad$ De acuerdo $\square \quad$ Indiferente $\square \quad$ En desacuerdo $\square \quad$ Muy en desacuerdo $\square$ 10. ¿Estas en líneas generales satisfecho con la asignatura de manera global?.

Muy de acuerdo $\square \quad$ De acuerdo $\square \quad$ Indiferente $\square \quad$ En desacuerdo $\square \quad$ Muy en desacuerdo $\square$ 